\title{
Increased systemic oxidatively generated DNA and RNA damage in schizophrenia
}

Jørgensen, Anders; Brødbæk, Kasper; Fink-Jensen, Anders; Knorr, Ulla; Greisen Søndergaard, Mia; Henriksen, Trine; Weimann, Allan; Jepsen, Peter; Lykkesfeldt, Jens; Poulsen, Henrik Enghusen; Jørgensen, Martin Balslev

Published in:

Psychiatry Research

DOI:

10.1016/j.psychres.2013.01.033

Publication date:

2013

Document version

Early version, also known as pre-print

Citation for published version (APA):

Jørgensen, A., Brødbæk, K., Fink-Jensen, A., Knorr, U., Greisen Søndergaard, M., Henriksen, T., Weimann, A., Jepsen, P., Lykkesfeldt, J., Poulsen, H. E., \& Jørgensen, M. B. (2013). Increased systemic oxidatively generated DNA and RNA damage in schizophrenia. Psychiatry Research, 209(3), 417-423.

https://doi.org/10.1016/j.psychres.2013.01.033 


\title{
Increased systemic oxidatively generated DNA and RNA damage in schizophrenia
}

\author{
Anders Jorgensen $^{\mathrm{a}, \mathrm{b}, *}$, Kasper Broedbaek ${ }^{\mathrm{c}, \mathrm{d}}$, Anders Fink-Jensen ${ }^{\mathrm{a}, \mathrm{b}, \mathrm{e}}$, Ulla Knorr ${ }^{\mathrm{a}}$, \\ Mia Greisen Soendergaard ${ }^{\mathrm{a}}$, Trine Henriksen ${ }^{\mathrm{c}, \mathrm{d}}$, Allan Weimann ${ }^{\mathrm{c}, \mathrm{d}}$, Peter Jepsen ${ }^{\mathrm{a}}$, \\ Jens Lykkesfeldt ${ }^{\mathrm{e}}$, Henrik Enghusen Poulsen ${ }^{\mathrm{c}, \mathrm{d}, \mathrm{e}}$, Martin Balslev Jorgensen ${ }^{\mathrm{a}, \mathrm{b}, \mathrm{e}}$ \\ ${ }^{a}$ Psychiatric Centre Copenhagen, University Hospital of Copenhagen, Denmark \\ ${ }^{\mathrm{b}}$ Laboratory of Neuropsychiatry, Department of Neuroscience and Pharmacology, University of Copenhagen, Denmark \\ c Laboratory of Clinical Pharmacology Q7642, Rigshospitalet, Copenhagen, Denmark \\ d Department of Clinical Pharmacology, Bispebjerg Hospital, Copenhagen, Denmark \\ e Faculty of Health and Medical Sciences, University of Copenhagen, Denmark
}

\section{A R T I C L E I N F O}

\section{Article history:}

Received 30 July 2012

Received in revised form

14 December 2012

Accepted 26 January 2013

\section{Keywords:}

Schizophrenia

Oxidative stress

8-oxodG

8-oxoGuo

Perceived stress

Cortisol

\begin{abstract}
A B S T R A C T
Schizophrenia is associated with a substantially increased somatic morbidity and mortality, which may partly be caused by accelerated cellular aging. Oxidative stress is an established mediator of aging and a suggested aetiological mechanism in both schizophrenia and age-related medical disorders such as cardiovascular disease, type 2 diabetes and dementia. We determined the urinary excretion of markers of systemic Deoxyribonucleic Acid (DNA) and Ribonucleic Acid (RNA) oxidation, 8-oxo-7,8-dihydro2'-deoxyguanosine and 8-oxo-7,8-dihydroguanosine, respectively, in 40 schizophrenia patients and 40 age- and sex-matched controls, using ultra-performance liquid chromatography with tandem mass spectrometry. Measures of psychopathology, perceived stress and cortisol secretion were collected. Patients were re-examined after four months. We found a $20 \%$ increase in the median excretion of both markers in schizophrenia patients vs. healthy controls $(P=0.003$ and $<0.001$, respectively). This difference persisted after the adjustment for multiple demographical, lifestyle and metabolic factors. In patients, the marker excretion was not influenced by medication load, and was not driven by symptom severity, perceived stress or cortisol secretion, neither at baseline nor in relation to changes at follow-up. We conclude that schizophrenia is associated with increased systemic nucleic acid damage from oxidation, which could constitute a molecular link between schizophrenia and its associated signs of accelerated aging.
\end{abstract}

(c) 2013 Elsevier Ireland Ltd. All rights reserved.

\section{Introduction}

Schizophrenia has been suggested to constitute a "syndrome of accelerated aging" (Kirkpatrick et al., 2008). Life expectancy is shortened with as much as 15-20 years, mainly due to increased mortality from cardiovascular disease (Hoang et al., 2011; Wahlbeck et al., 2011). Furthermore, schizophrenia is associated with mildly accelerated cognitive decline across the lifespan, paralleled by structural brain alterations such as ventricular enlargement and hippocampal volume reduction (Jeste et al., 2011; Shenton et al., 2001; Velakoulis et al., 2006). Finally, some studies have found telomere length, a molecular correlate of aging, to be reduced in schizophrenia patients in comparison to

\footnotetext{
* Corresponding author at: Psychiatric Centre Copenhagen, University Hospital of Copenhagen, Rigshospitalet, Blegdamsvej 9, DK-2100 Copenhagen, Denmark. Tel.: +45 5320 6212; fax: +4535456238.

E-mail address: anders.01.joergensen@regionh.dk (A. Jorgensen).
}

healthy controls (Kao et al., 2008), although others have found no difference (Mansour et al., 2011).

Recent evidence has converged on mitochondrial dysfunction and oxidative stress as potential pathogenetic mechanisms in schizophrenia, with findings of both cerebral and peripheral perturbations in these systems (Arvindakshan et al., 2003b; Gysin et al., 2007; Kunz et al., 2008; Prabakaran et al., 2004; Regenold et al., 2009; Rosenfeld et al., 2011), as well as beneficial clinical effects of antioxidant therapy (Arvindakshan et al., 2003a; Chengappa et al., 2012; Dakhale et al., 2005). Similarly, administration of N-acetyl cystein (NAC) - a precursor of the key brain antioxidant glutathione - lead to improvement of clinical symptoms and auditory sensory processing in schizophrenia (Berk et al., 2008; Lavoie et al., 2007). Interestingly, preclinical experiments have indicated that glutathione deficits produce neuroanatomical and cognitive deficits similar to those observed in schizophrenia, and that these effects are counteracted by NAC administration (Cabungcal et al., 2012; Choy et al., 2010). 
Oxidative stress on cellular constituents such as Deoxyribonucleic Acid (DNA) and Ribonucleic Acid (RNA) is suspected to play a critical role in cellular aging (Finkel and Holbrook, 2000), as well as in the development of age-related conditions such as type 2 diabetes, cardiovascular disease and neurodegeneration (BossyWetzel et al., 2004; Broedbaek et al., 2011; Harrison et al., 2003; Nunomura et al., 2009). Genotoxic stress from oxidation activate DNA damage signalling pathways and accelerates telomere erosion, thereby increasing the risk of cellular senescence or apoptosis, which are known to be key events in the aging process (Sahin and DePinho, 2010). Hence, a chronic state of increased oxidative stress on nucleic acids could perhaps underlie the association between schizophrenia, cognitive decline and ageassociated morbidity. In that context, a recent study provided compelling evidence that mitochondrial dysfunction and oxidative stress may be specifically involved in the cognitive deficit endophenotype of schizophrenia (Jablensky et al., 2012).

Urinary 8-oxo-7,8-dihydro-2'-deoxyguanosine (8-oxodG) and 8-oxo-7,8-dihydroguanosine (8-oxoGuo) are biomarkers of systemic oxidative stress on DNA and RNA, respectively. Urinary 8-oxodG has been extensively validated as a marker of oxidatively generated DNA damage through a number of studies (Deng et al., 1998; Evans et al., 2010b; Loft et al., 1995) The experimental induction of wide-spread DNA damage by an exogenous carcinogen lead to the transient accumulation of 8-oxodG in nuclear DNA of the bone marrow, liver and kidneys, and a corresponding increase in urinary 8-oxodG excretion in rats (Deng et al., 1998). Circulating 8-oxodG is completely cleared through renal excretion, and the urinary excretion of 8-oxodG thereby reflects the overall whole-body oxidative stress on DNA (Evans et al., 2010b; Loft et al., 1995). Although similar validation data on 8-oxoGuo are not available, the two markers are usually closely correlated (Joergensen et al., 2011), and thus probably reflect the same whole-body oxidative stress on nucleic acids.

For this study, we determined the urinary excretion of 8-oxodG and 8-oxoGuo in forty medicated schizophrenia patients and forty age- and sex-matched controls. We further correlated baseline and 4-month follow-up 8-oxodG/8-oxoGuo excretion to measures of perceived psychological stress and cortisol secretion, which have previously been linked to telomere attrition and nucleic acid oxidation (Epel et al., 2004; Joergensen et al., 2011). The urinary content of 8 -oxodG/8-oxoGuo was assayed by a highly specific and sensitive mass spectrometric detection (Henriksen et al., 2009). The method has been extensively validated, including in a recent international, inter-laboratory collaboration (Evans et al., 2010a).

We hypothesized an increased excretion of nucleic acid oxidation markers in schizophrenia patients vs. controls, and a positive correlation between perceived stress/cortisol secretion and the marker excretion, both at baseline as well as in relation to changes over a 4 month period.

\section{Methods}

\subsection{General study outline}

Recruitment took place from September 2008 through May 2011. Patients were recruited by referral from doctors at the Psychiatric Centre Copenhagen, which provides mental health services to the citizens of the central, northern and north-western area of Copenhagen. Physicians at the centre were informed of the study in writing and by word of mouth, and received regular reminders to refer patients. Both inpatients as well as patients from the affiliated outpatient clinics were recruited. Investigations were carried out at baseline ("T0") and repeated after 4 months ("T4"). In the intermediate period, patients received treatment as usual, including medication.

The inclusion criterion for patients was an ICD-10 diagnosis of either schizophrenia or acute schizophreniform psychosis, confirmed by a structured interview at referral. Exclusion criteria were: (1) somatic disease and somatic medication. A non-regular use of e.g. painkillers or asthma medication was allowed, (2) abuse of alcohol, marihuana or other drugs of abuse, (3) coercion of any kind, (4) severely disorganised thinking, making it impossible to obtain an informed consent, (5) use of dietary supplements, and (6) pregnancy or breast-feeding. Of forty-five patients referred to the study and accepting to participate, 40 were included. In the rejected patients, the diagnosis of schizophrenia was considered uncertain after the inclusion interview $(N=4)$, or the biochemical screening revealed a medical disorder $(N=1)$.

Healthy controls were recruited from the blood donation corps at Rigshospitalet by personal contact, as they were scheduled for donating blood. Exclusion criteria for the healthy controls were: (1) any psychiatric or somatic disease (2) abuse of alcohol, marihuana or other drugs, (3) use of any medication including dietary supplements, and (4) first degree family members with psychiatric disease. A total of 175 healthy controls meeting none of the exclusion criteria were asked to participate, and of these, 40 accepted to be included. They underwent the same examinations as the patients (except for schizophreniform psychopathology), but were only examined at T0.

The study schedule was as follows: on day 1, blood was drawn in the fasting state at $9 \mathrm{AM}$. In a few cases (two controls and six patients), blood was not drawn in the morning, and these cases were omitted from analyses including plasma cortisol. A spot urine sample was obtained from the first voided urine after blood sampling. Sociodemographic and clinical data were recorded. Ratings and questionnaires were completed. On day 2 (or no more than one week from the examination), the participant made salivary samples for cortisol determination. At $\mathrm{T} 4$, the sequence was repeated.

The study protocol complied with the Declaration of Helsinki, and was approved by the Regional Commitee on Research Ethics (H-D-2008-064) and the National Data Protection Agency (2008-41-2052). All participants gave a written informed consent before inclusion.

\subsection{Interviews and questionnaires}

A Schedules in Clinical Neuropsychiatry (SCAN)-interview (Wing et al., 1990) was applied at T0 to ensure that the ICD-10 diagnostic criteria for schizophrenia (F20.0-F20.9) were met, and that no lifetime psychiatric morbidity was present in the healthy controls. Medical doctors with previous clinical experience in psychiatry performed all the SCAN ratings. The severity of psychopathology was measured with the Positive and Negative Syndrome Scale (PANSS) (Kay et al., 1987). Regular co-ratings with a certified expert PANSS-rater (AFJ) were performed, yielding an Intraclass Correlation Coefficient of 0.91 for positive items, 0.76 for negative items, 0.79 for general items and 0.83 for all items, indicating very good agreement across all subscales. The level of perceived stress was assessed with the Perceived Stress Scale 10-item (PSS) (Cohen and Williamson, 1988).

\subsection{General biochemical screening}

This comprised a full blood count, hepatic enzymes, total cholesterol, lowdensity lipoprotein (LDL) and high-density lipoprotein (HDL) cholesterol, triglycerides, C-reactive protein (CRP), thyroid stimulating hormone (TSH), sodium, potassium, creatinine, glucose, glycated haemoglobin $(\mathrm{HbA} 1 \mathrm{c})$, and plasma cortisol.

\subsection{Urinary 8-oxodG and 8-oxoGuo}

The urinary sample was obtained with a standard sampling kit without any additives. Samples were kept on ice and transferred within hours to storage at $-20{ }^{\circ} \mathrm{C}$ until analysis. The urinary content of the oxidatively modified guanine nucleosides was assayed using ultraperformance liquid chromatography and tandem mass spectrometry (UPLC-MS/MS). The chromatographic separation was performed on an Acquity UPLC system (Waters, Milford, MA, USA). The column used was an Acquity UPLC BEH Shield RP18 column $\left(1.7 \mu \mathrm{m}, 2.1 \times 100 \mathrm{~mm}^{2}\right)$ protected with in-line filter $\left(4 \times 2 \mathrm{~mm}^{2}, 0.2 \mu \mathrm{m}\right)$ both obtained from Waters. The MS detection was performed on an API 3000 triple quadrupole mass spectrometer (Sciex, Toronto, Canada) equipped with an ESI ion source (Turbospray) operated in the positive mode. Details of the analysis are described elsewhere (Henriksen et al., 2009). The urinary creatinine concentration was determined by Jaffe's reaction. The 8-oxodG/8-oxoGuo excretion is defined as the urinary concentration of the nucleoside normalised to urinary creatinine concentration (Evans et al. 2010a)

\subsection{Plasma markers}

Plasma was obtained from EDTA-coated tubes which were centrifuged at $4{ }^{\circ} \mathrm{C}$ and $1590 \mathrm{~g}$ for $10 \mathrm{~min}$. Samples were stored at $-80{ }^{\circ} \mathrm{C}$ within $30 \mathrm{~min}$ from venipuncture. Malondialdehyde (MDA, a marker of lipid peroxidation), as well as 
Table 1

Basic data of healthy controls and schizophrenia patients at baseline. Numbers are in mean ( \pm standard deviation) or median (interquartile range).

\begin{tabular}{|c|c|c|c|}
\hline & Control $(N=40)$ & Schizophrenia $(N=40)$ & $P$-value \\
\hline Gender (M/F) & $20 / 20$ & $20 / 20$ & 1.0 \\
\hline Age (years) & $31.4( \pm 9.8)$ & $33.0( \pm 10.7)$ & 0.49 \\
\hline Ethnicity (Caucasian/other) & $40 / 0$ & $37 / 3$ & 0.21 \\
\hline Education (years) ${ }^{a}$ & $4(3-5)$ & $3(0-3)$ & 0.003 \\
\hline Smoker (\%) & 21 & 55 & 0.002 \\
\hline Cigarettes per day (if smoker) & $8.1( \pm 6.4)$ & $18.1( \pm 11.3)$ & 0.03 \\
\hline Alcohol (drinks per week) & $5(2-10)$ & $1(0-5)$ & 0.002 \\
\hline Exercise (hours per week) & $3(1-5)$ & $0(0-2)$ & $<0.001$ \\
\hline Oral contraceptives (number of users) & 1 & 5 & $<0.001$ \\
\hline Body Mass Index & $25.1( \pm 3.8)$ & $27.2( \pm 5.7)$ & 0.06 \\
\hline Waist-hip ratio & $0.80( \pm 0.07)$ & $0.89( \pm 0.08)$ & $<0.001$ \\
\hline Systolic blood pressure (mmHg) & $124.6( \pm 11.6)$ & $127.1( \pm 12.2)$ & 0.34 \\
\hline Diastolic blood pressure ( $\mathrm{mmHg}$ ) & $78.9( \pm 8.6)$ & $78.5( \pm 7.3)$ & 0.37 \\
\hline Pulse $\left(\min ^{-1}\right)$ & $64.6( \pm 10.6)$ & $81.0( \pm 14.3)$ & $<0.001$ \\
\hline Total cholesterol $(\mathrm{mmol} / \mathrm{L})$ & $4.7( \pm 0.8)$ & $4.9( \pm 0.9)$ & 0.44 \\
\hline HDL cholesterol (mmol/L) & $1.5( \pm 0.4)$ & $1.4( \pm 0.4)$ & 0.40 \\
\hline LDL cholesterol (mmol/L) & $2.8( \pm 0.6)$ & $2.8( \pm 0.8)$ & 0.75 \\
\hline Triglycerides (mmol/L) & $0.90(0.70-1.15)$ & $1.25(0.76-2.00)$ & 0.02 \\
\hline Glucose $(\mathrm{mmol} / \mathrm{L})$ & $5.2( \pm 0.4)$ & $5.1( \pm 0.6)$ & 0.48 \\
\hline $\mathrm{HbA} 1 \mathrm{c}(\mathrm{mmol} / \mathrm{L})$ & $5.2( \pm 0.3)$ & $5.4( \pm 0.4)$ & 0.01 \\
\hline Plasma Creatinine $(\mu \mathrm{mol} / \mathrm{L})$ & $73( \pm 16)$ & $69( \pm 14)$ & 0.20 \\
\hline Urine Creatinine $(\mathrm{mmol} / \mathrm{L})$ & $12.3( \pm 6.3)$ & $13.9( \pm 8.4)$ & 0.34 \\
\hline $\mathrm{CRP}(\mathrm{mg} / \mathrm{L})$ & $1(1-2)$ & $2(1-4)$ & 0.002 \\
\hline $\mathrm{TSH}\left(\times 10^{-3} \mathrm{IU} / \mathrm{L}\right)$ & $1.65(1.16-2.27)$ & $1.80(1.46-2.73)$ & 0.04 \\
\hline Plasma cortisol (nmol/L) & $442(310-525)$ & $494(394-666)$ & 0.03 \\
\hline Plasma vitamin $C(\mu \mathrm{mol} / \mathrm{L})$ & $61.8( \pm 14.1)$ & $53.7( \pm 23.6)$ & 0.07 \\
\hline Plasma uric acid $(\mu \mathrm{mol} / \mathrm{L})$ & $245( \pm 52)$ & $237( \pm 56)$ & 0.53 \\
\hline
\end{tabular}

Data are analysed with $t$-test, Mann-Whitney test or Chi-squared test, as appropriate.

Abbreviations: HDL: high-density lipoprotein. LDL: low-density lipoprotein. HbA1c: glycated haemoglobin.

CRP: C-reactive protein. TSH: thyroid stimulating hormone.

${ }^{\text {a }}$ Years of completed education beyond Danish compulsory school (9 years).

the two plasma antioxidants uric acid and vitamin $\mathrm{C}$ were also determined, using previously described chromatographic methods (Lykkesfeldt, 2001, 2007a).

\subsection{Salivary cortisol}

Samples were obtained by the "Salivette" system immediately upon awakening, at 15, 30, 45 and 60 min after awakening, at 6 PM and at 11 PM. Samples were made either in the participant's own home and mailed to the laboratory (controls and out-patients) or at the ward (in-patients). Salivary cortisol is preserved for weeks at room temperature (Kirschbaum and Hellhammer, 1994). Participants were instructed to keep the Salivette cotton wad in the mouth for $>1 \mathrm{~min}$, and were not allowed to eat, smoke, brush his teeth or drink (other than small amounts of water) 30 min before each sample. Participants were given a form on which they noted the time they fell to sleep the night before sampling, woke up on the morning of the sampling and the time they made every sample. Upon receipt at the laboratory, the Salivette tubes were centrifugated at $1590 \mathrm{~g}$ for 5-10 min to extract the saliva. Samples were stored at $-80{ }^{\circ} \mathrm{C}$ until analysis.

Saliva was assayed for cortisol by an electrochemiluminescence immunoassay, using a commercially available kit (Roche Diagnostics GmbH, Mannheim, Germany). The lower detection limit of the assay was $1 \mathrm{nmol} / \mathrm{L}$. The inter- and intra-assay coefficients of variation of the assay of were $7.1 \%$ and $4.0 \%$, respectively, according to the manufacturer. The area under the curve with respect to the ground level for all samples (AUCg) were calculated for each participant by the trapezoidal rule (Pruessner et al., 2003), which incorporates cortisol values and the exact times between samples to estimate whole-day "exposure" to unbound cortisol. Before computations, extreme values in each group for each time point (outside the 99th percentile) were excluded (30 out of a total of 658 determinations). For series of samples with more than one sample missing, the AUC was not computed. If only one sample was missing, values were replaced by the mean of the two adjacent values, or, if the missing value were either the awakening or 11 PM sample, by the mean of the full sample for that time point. We were able to compute AUCg for 31 controls and 29 patients at T0, and for 22 patients at T4. In both groups, cortisol concentrations displayed the expected diurnal pattern (peak values at 30 min after awakening and nadir at $11 \mathrm{PM}$ ).

All samples from each participant were analysed in the same batch, but control and patient samples were analysed on separate occasions. Therefore, we refrained from making direct comparisons between patients and controls, and only analysed the correlations with 8-oxodG/8-oxoGuo in each group separately.

\subsection{Statistics}

All data are presented as means ( \pm standard deviation) or median (interquartile range). Normal distributed data were analysed with independent samples $t$-tests, analysis of variance (ANOVA) or paired samples $t$-tests as relevant, and non-normal distributed data with Mann-Whitney test. For categorial data, Chi-squared tests were used. For the analysis of 8-oxodG/8-oxoGuo, Pearson correlations and two multiple linear regression models were applied. In model 1, the independent variables were demographical and lifestyle factors potentially associated with oxidative stress (age, gender, ethnicity, smoking, alcohol intake, exercise and oral contraceptive use). Model 2 additionally included as independent variables all the clinical and metabolic factors showing differences between the groups (waist-hip ratio, pulse, triglycerides, HbA1c, CRP, TSH and cortisol), as well as the plasma antioxidants (Table 1). For all parametric tests, the dependent variables in question deviating from normal distribution (8-oxodG/8-oxoGuo and plasma cortisol) were transformed by the natural logarithm. All statistical analyses were performed using the Statistical Package for the Social Sciences (SPSS) software version 20.0 (IBM Corporation, NY, USA). Statistical significance was defined as $P<0.05$. All statistical tests were two sided.

\section{Results}

Basic data of patients and controls are presented in Table 1. The urinary excretion of both 8-oxodG and 8-oxoGuo was significantly increased in the schizophrenia vs. the control group (8-oxodG: $1.7(1.4-2.4)$ vs. $1.4(1.2-1.6) \mathrm{nmol} / \mathrm{mmol}$ urinary creatinine, Mann-Whitney $U$-test, Mann-Whitney $U(\mathrm{MWU})=$ 496.0, $P=0.003$. 8-oxoGuo: $2.2(1.9-2.7)$ vs. $1.8(1.4-2.1) \mathrm{nmol} /$ mmol, MWU $=348.0, P<0.001$ ) (Fig. 1). The effect size (Cohen's $d$ ) was 0.69 for 8 -oxodG and 1.13 for 8 -oxoGuo, indicating a moderate and large effect, respectively. The median excretion of 8 -oxoGuo was $29 \%$ higher than 8 -oxodG in both groups, and the two markers were highly correlated (Pearson's $r=0.52, P<0.001$ ). In contrast to the nucleic acid oxidation markers, MDA did not 

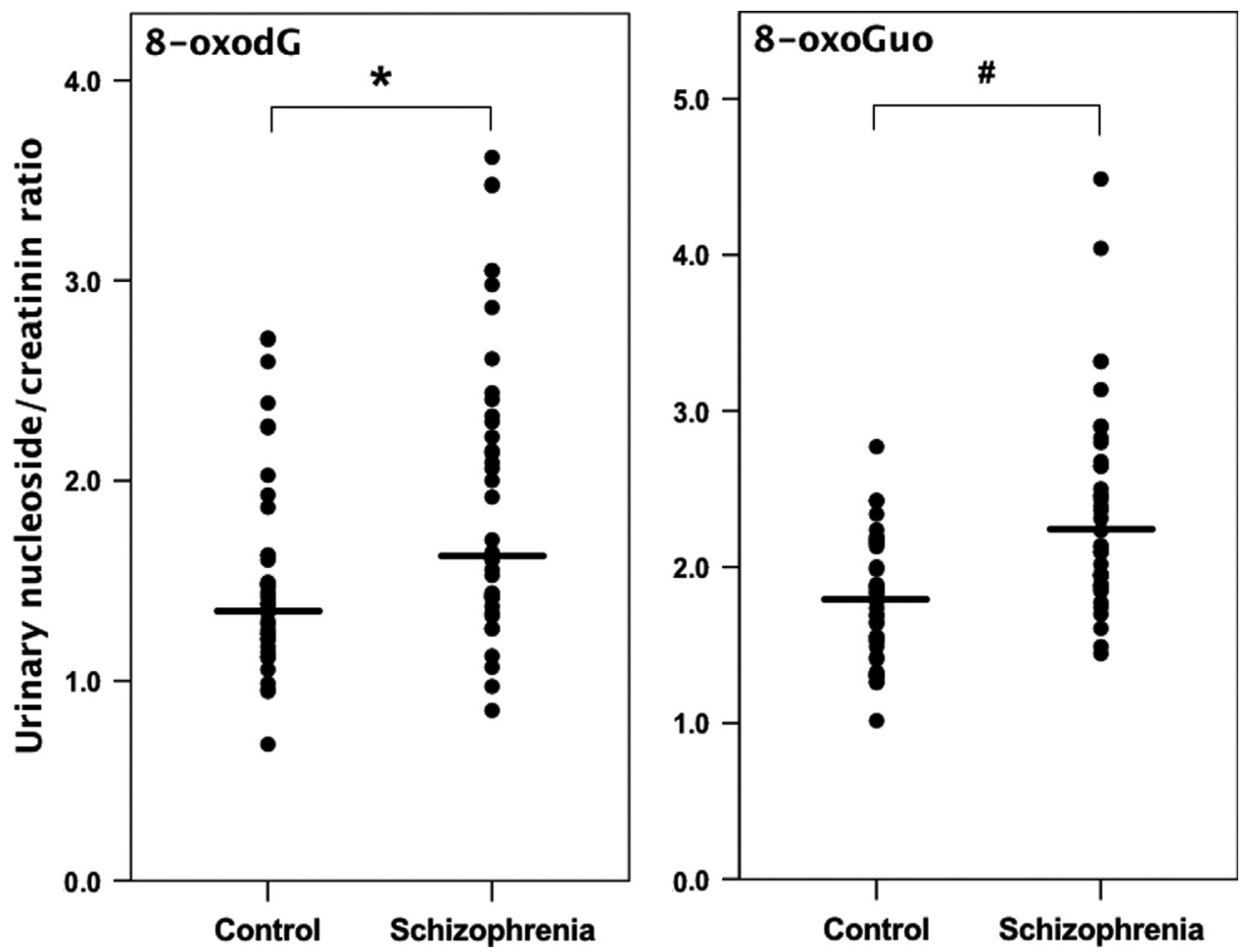

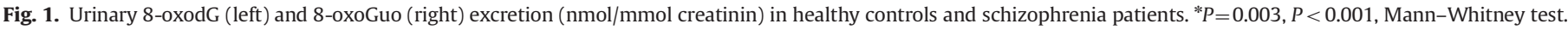

significantly differ between the groups (controls: $0.89( \pm 0.23)$ $\mu \mathrm{mol} / \mathrm{L}$, schizophrenia: $0.78( \pm 0.33) \mu \mathrm{mol} / \mathrm{L}$, independent samples $t$-test, $t=1.53$, d.f. $=74, P=0.13)$.

The difference in 8-oxodG/8-oxoGuo excretion between the groups persisted after the adjustment for demographical and lifestyle variables (Model 1) (8-oxodG: $\beta=0.52, P<0.001$. 8-oxoGuo: $\beta=0.68, P<0.001)$. After the additional adjustment for multiple clinical and metabolic factors (model 2), group was still significantly associated with 8-oxodG/8-oxoGuo excretion (8oxodG: $\beta=0.61, P=0.002$. 8 -oxoGuo: $\beta=0.64, P=0.002$ ). In a separate analysis, we found no difference in neither marker between smokers vs. non-smokers in neither the control (8oxodG: $M W U=99.0, P=0.33$. 8-oxoGuo: $M W U=98.0, P=0.31$ ) nor in the patient group (8-oxodG: $M W U=146.0, P=0.16$. 8-oxoGuo: $M W U=160.0, P=0.3$ ).

The clinical baseline characteristics of schizophrenia patients are shown in Table 2. All patients received second generation antipsychotics (SGA). The primary antipsychotic agent used was risperidone $(N=9)$, quetiapine $(N=9)$, aripiprazole $(N=6)$, olanzapine $(N=5)$ or clozapine $(N=5)$. Six patients received other SGAs. None of the markers showed associations with the number of antipsychotics used (8-oxodG: one-way ANOVA, $F(2,37)=0.52$, $P=0.60$, 8-oxoGuo: $F(2,37)=1.26, P=0.30)$, the Defined Daily Dose (DDD) of antipsychotics (8-oxodG: Pearson's $r=-0.18, P=0.26$, 8-oxoGuo: $r=0.11, P=0.5$ ) or the total DDD of all medications (8-oxodG: $r=-0.26, P=0.1,8$-oxoGuo: $r=0.06, P=0.73$ ). The individual agents were not associated with significant differences in the marker excretion, except for a higher 8-oxodG excretion in the five clozapine users compared to risperidone users (one-way ANOVA, $F(5,34)=3.3, P=0.02$, post-hoc Tukey test of clozapine vs. risperidone: $P=0.006$ ). The 8 -oxodG/8-oxoGuo excretion was not associated with the duration of illness (even after adjusting for age), number of admissions, or admission status (in- or out-patient) at the time of inclusion.

As expected, patients had substantially higher perceived stress scores than healthy controls $(22.3( \pm 6.7)$ vs. $7.7( \pm 4.2)$ points,
Table 2

Clinical characteristics of schizophrenia patients at baseline. Numbers are in mean ( \pm standard deviation) or median (interquartile range).

\begin{tabular}{ll}
\hline Diagnosis, ICD-10 & \\
Paranoid schizophrenia & $55 \%$ \\
Undifferentiated schizophrenia & $28 \%$ \\
Hebephrenic schizophrenia & $12 \%$ \\
Unspecified schizophrenia & $5 \%$ \\
Disease course & \\
Age of onset (years) & $23.0( \pm 5.3)$ \\
Duration of illness (months) & $76(36-189)$ \\
Number of admissions & $3(1-6)$ \\
In-patient at inclusion & $60 \%$ \\
Medication & \\
Duration of current antipsychotic treatment (months) & $20(3-68)$ \\
Number of antipsychotics used & \\
$\quad$ One & $77 \%$ \\
$\quad$ Two or three & $23 \%$ \\
Antipsychotics, Defined Daily Dose & \\
Antidepressant use & $1.5(0.7-2.3)$ \\
If antidepressant use, Defined Daily Dose & $43 \%$ \\
Benzodiazepine use & $1.4(1.0-2.0)$ \\
If benzodiazepine use, Defined Daily Dose & $28 \%$ \\
Psychopathology & $0.6(0.3-1.2)$ \\
PANSS positive score & \\
PANSS negative score & $22.2( \pm 4.3)$ \\
PANSS general score & $22.3( \pm 5.6)$ \\
PANSS total score & $44.6( \pm 8.5)$ \\
& $89.1( \pm 16.5)$
\end{tabular}

a All patients received second-generation antipsychotics. Apart from the listed medications, three patients received anticholinergic agents, two patients received antiepileptics and one patient received electroconvulsive therapy.

${ }^{\mathrm{b}}$ As defined by the World Health Organization.

independent samples $t$-test, $t=-12.1$, d.f. $=78, P<0.001$ ). There were no significant associations between PSS scores and the nucleic acid oxidation markers in neither the control nor the schizophrenia group. Neither the total nor any subscale PANSS scores of schizophrenia patients correlated with either of the markers. 


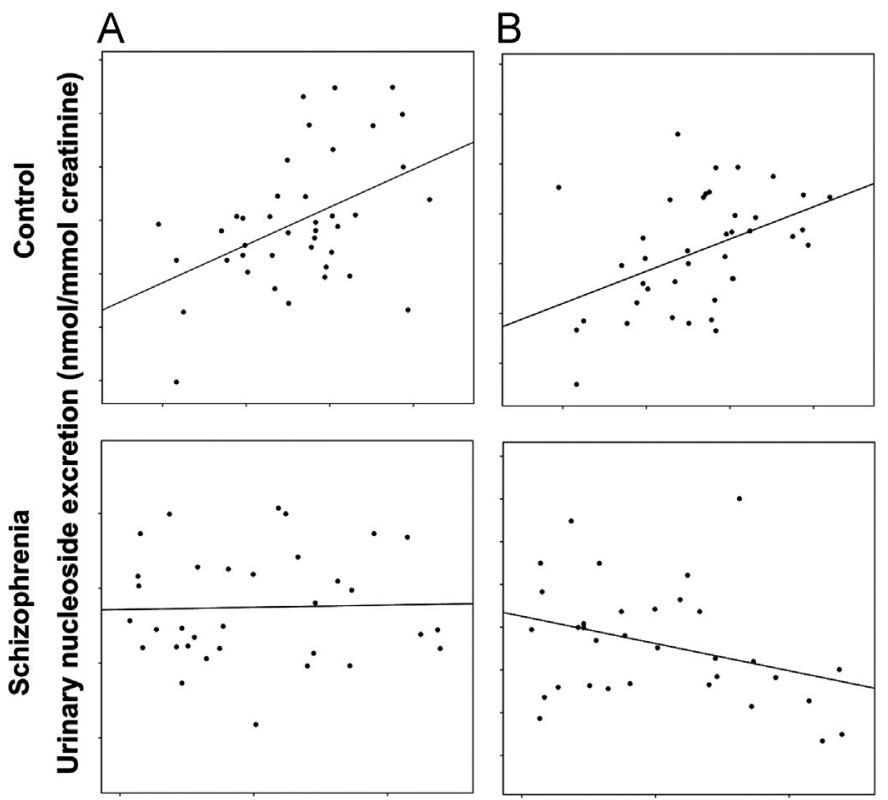

Plasma cortisol
C D

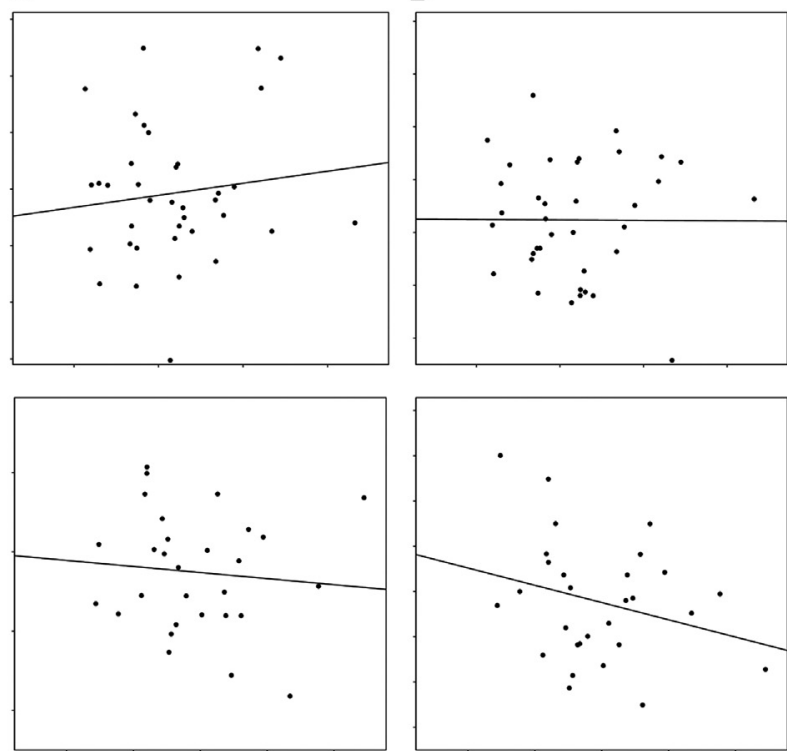

AUC salivary cortisol

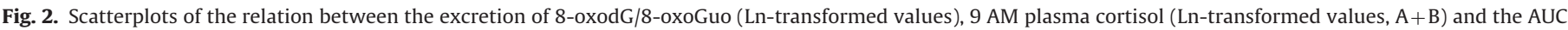

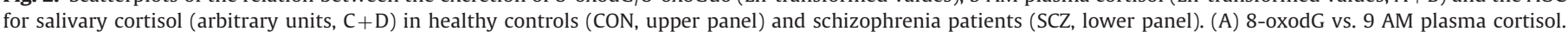

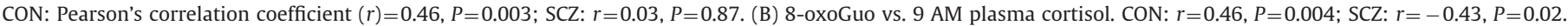

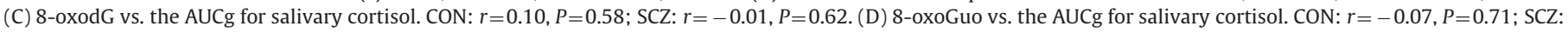
$r=-0.27, P=0.16$.

In the control group, both markers were positively associated with the log-transformed 9 AM plasma cortisol, while being nonassociated or significantly negatively associated with the plasma cortisol in the schizophrenia group. For the correlation to AUCg, a similar pattern of a more negative slope in the schizophrenia group was seen (results are summarized in Fig. 2). There were no significant correlations between plasma MDA and the cortisol measures in neither group (data not shown).

Eighty per cent of the included patients completed the study at T4. Of the non-completers, four refused to participate, two could not be contacted, and two had developed somatic disease. There were no significant differences between the completers and the non-completers with respect to age (independent samples $t$-test, $t=-1.12$, d.f. $=38, P=0.91$ ), DDD of antipsychotic medication $(t=-0.7$, d.f. $=38, P=0.49)$, or total PANSS score $(t=-0.45$, d.f. $=38, P=0.65)$. DDDs of SGAs or all medications were not changed from T0 to T4 (DDD, SGA: Wilcoxon Signed Ranks test, $Z=-0.2, P=0.84$. DDD, all medications: $Z=-0.3, P=0.77$ ).

The T4 median excretion of 8 -oxodG/8-oxoGuo continued to be elevated in comparison to the T0 levels of healthy controls (8-oxodG: Mann-Whitney $U$-test, $M W U=460.0, P=0.04$, 8-oxoGuo: $M W U=$ 347.0, $P=0.001$ ), reflecting a strong intra-individual correlation between T0 and T4 values (8-oxodG: Pearson's $r=0.62, P<0.001$. 8-oxoGuo: $r=0.58, P=0.001)$. We found no associations between T0 and T4 changes in 8-oxodG/8-oxoGuo excretion vs. changes in PANSS (range: $-28-+23$ points), PSS, or cortisol secretion.

\section{Discussion}

Only a few studies have examined levels of DNA damage in schizophrenia. Two previous studies did not find elevated lymphocyte DNA strand breaks, as measured by the comet assay, compared to controls (Psimadas et al., 2004; Young et al., 2007). A recent study found increased levels of phosphorylated histone variant $\mathrm{H} 2 \mathrm{AX}$, an index of DNA double strand breaks, in cultured lymphoblasts from schizophrenia patients compared to healthy controls, and an attenuated activation of the same marker after cell irradiation in patients (Catts et al., 2012). In a post-mortem study, an increased intra-neuronal 8-oxodG immunoreactivity in the hippocampus of elderly, poor-outcome schizophrenia patients was observed (Nishioka and Arnold, 2004). However, to our knowledge, this study provides the first evidence for increased systemic DNA and RNA oxidation in vivo in schizophrenia. In our cohort, the median urinary excretion of both 8-oxodG and 8 -oxoGuo was approximately $20 \%$ higher in the schizophrenia patients, and the difference persisted after the adjustment for multiple confounders of oxidative stress, indicating that the increased nucleic acid oxidation in patients is not merely an epiphenomenon of lifestyle or dysmetabolism.

Although the data by no means proves a causal relationship between schizophrenia and oxidatively generated DNA/RNA damage, the finding is not inconsistent with the hypothesis that oxidative stress is a pathophysiological mechanism in schizophrenia. In contrast to the finding of others (Arvindakshan et al., 2003b; Zhang et al., 2006), plasma markers of lipid peroxidation and antioxidant status did not differ between the groups. This discrepancy could be due to methodological issues, in that the above-mentioned studies determined MDA by the Thiobarbituric Acid assay, whereas we used HPLC, which is considered a more specific method for the measurement of MDA (Lykkesfeldt, 2007b). Our finding could indicate a specific susceptibility to nucleic acid oxidation in schizophrenia. The mitochondria are the primary source of ROS, and disturbances of mitochondrial function will lead to increased formation of ROS and oxidative stress (Turrens, 2003). If mitochondrial perturbations are a core feature of the biology of schizophrenia as suggested by others (Jablensky et al., 2012; Prabakaran et al., 2004; Rosenfeld et al., 2011), then molecules located in the proximity of the mitochondria, such as RNA and mitochondrial DNA, could be speculated to be particularly exposed to oxidative damage, whereas markers of extracellular oxidative processes such as plasma MDA would be relatively unaffected. 
The markers remained elevated at a 4-month follow-up examination in the patients, reflecting a strong correlation between intra-individual T0 and T4 values, and were not correlated to the duration of illness or any measure of current severity of disease. This indicates that the elevated nucleic acid damage from oxidation is a "trait" rather than a "state" phenomenon, possibly due to a genetically determined increased oxidative stress, as suggested by others (Tosic et al., 2006), or as a result of more persistent and stable metabolic changes developing early in the disease course.

Our finding could have implications for the signs of accelerated aging observed in schizophrenia. Genotoxic stress caused by oxidants is recognised as a critical event in cellular aging (Finkel and Holbrook, 2000). The oxidatively generated damage to DNA accumulates with age and may induce cell senescence or apoptosis (Moller et al., 2010; Sahin and DePinho, 2010). Furthermore, an increasing attention to RNA oxidation as an instigator of human aging and neuropathology is emerging (Nunomura et al., 2009). Interestingly, a recent post-mortem study found increased oxidation of RNA, but not of DNA, in the hippocampus of schizophrenia patients (Che et al., 2010). Similarly, we found a more pronounced difference in the RNA marker excretion (effect size (Cohen's $d)=1.13$ ) compared to the DNA marker excretion (Cohen's $d=0.69$ ) in patients vs. controls.

The UPLC-MS/MS method for the detection of urinary nucleosides has been extensively validated and allows for a high throughput analysis of samples obtained non-invasively (Evans et al., 2010a), and the determined excretion levels of 8-oxodG/ 8 -oxoGuo are within the reference ranges reported by others (Andreoli et al., 2011). Based on this, 8-oxodG/8-oxoGuo could have utility as biomarkers in larger-scale, prospective studies of aging and mortality in schizophrenia. As an illustration of this potential use, we recently found 8-oxoGuo (but not 8-oxodG) to be an independent predictor of long-term mortality in a large cohort of newly diagnosed type II diabetics (Broedbaek et al., 2011)

We did not find the expected association between the marker excretion and measures of cortisol secretion in schizophrenia patients. In contrast, and in replication of a previous finding (Joergensen et al., 2011), plasma cortisol were positively correlated to both markers in the control group. Corticosteroids impact on mitochondrial function (Du et al., 2009) and tissue DNA oxidation levels (Caro et al., 2007). The lack of an association or even a negative association - in the schizophrenia patients suggests that the cause of the increased nucleic acid oxidation lies outside of the neurohormonal stress response. It could be speculated that the chronic stress state of suffering from schizophrenia leads to a reduced cellular sensitivity to cortisol, as previously found in a non-pathological human stress condition (being caregiver for a cancer patient) (Miller et al., 2008).

There are several limitations to the study. (1) The crosssectional comparison of patients and controls does not allow for inferences on the mechanistic relationship between schizophrenia and oxidatively generated nucleic acid damage, and confounding factors which were not determined in the study could contribute to the finding. (2) The sample size is relatively low, and this may explain the null finding with regards to the association between 8-oxodG/8-oxoGuo and the psychometric variables (psychopathology and perceived stress). (3) We found no associations between the markers and any measure of medication load, and this is in line with evidence that only the first generation antipsychotics induce oxidative stress (Kropp et al., 2005). However, an effect of medication cannot entirely be ruled out, and the finding should be replicated in drug-naïve patients. (4) Likewise, while we found no effect of smoking on the markers in neither group, and the adjustment for smoking in multiple regression analysis did not alter the result, we can not rule out an effect of smoking habits, which differed substantially between the patient and control groups.

In conclusion, we found that schizophrenia was associated with a $20 \%$ increase in median urinary excretion of DNA and RNA oxidation markers compared to healthy controls. This difference remained significant after the adjustment for multiple sociodemographic, lifestyle, and metabolic variables. Although causal relations cannot be inferred from the study, increased rates of oxidatively generated nucleic acid damage could constitute a molecular link between schizophrenia and the signs of accelerated aging associated with this severe mental disorder.

\section{Acknowledgements}

The study was funded by the Psychiatric Centre Copenhagen and Grants from the Mental Health Services of the Capital Region of Denmark, The Ivan Nielsen Foundation, The Marie and Børge Krogh Foundation, M.D. Gerhard Linds Grant, The Psychiatric Research Foundation of 1967 and The Eli and Egon Larsen Foundation. We wish to thank all the participants. We further thank Drs. Jeanett Bauer and Lars Søndergaard for the referral of patients, and lab technician Allan Hansen for technical assistance.

\section{References}

Andreoli, R., Mutti, A., Goldoni, M., Manini, P., Apostoli, P., De Palma, G., 2011 Reference ranges of urinary biomarkers of oxidized guanine in (2'-deoxy) ribonucleotides and nucleic acids. Free Radical Biology and Medicine 50, 254-261.

Arvindakshan, M., Ghate, M., Ranjekar, P.K., Evans, D.R., Mahadik, S.P., 2003a Supplementation with a combination of omega-3 fatty acids and antioxidants (vitamins E and C) improves the outcome of schizophrenia. Schizophrenia Research 62, 195-204.

Arvindakshan, M., Sitasawad, S., Debsikdar, V., Ghate, M., Evans, D., Horrobin, D.F. Bennett, C., Ranjekar, P.K., Mahadik, S.P., 2003b. Essential polyunsaturated fatty acid and lipid peroxide levels in never-medicated and medicated schizophrenia patients. Biological Psychiatry 53, 56-64.

Berk, M., Copolov, D., Dean, O., Lu, K., Jeavons, S., Schapkaitz, I., Anderson-Hunt, M., Judd, F., Katz, F., Katz, P., Ording-Jespersen, S., Little, J., Conus, P., Cuenod, M. Do, K.Q., Bush, A.I., 2008. N-acetyl cysteine as a glutathione precursor for schizophrenia-a double-blind, randomized, placebo-controlled trial. Biological Psychiatry 64, 361-368.

Bossy-Wetzel, E., Schwarzenbacher, R., Lipton, S.A., 2004. Molecular pathways to neurodegeneration. Nature Medicine 10 (Suppl.), S2-S9.

Broedbaek, K., Siersma, V., Henriksen, T., Weimann, A., Petersen, M., Andersen, J.T. Jimenez-Solem, E., Stovgaard, E.S., Hansen, L.J., Henriksen, J.E., Bonnema, S.J., de Fine Olivarius, N., Poulsen, H.E., 2011. Urinary markers of nucleic acid oxidation and long-term mortality of newly diagnosed type 2 diabetic patients. Diabetes Care 34, 2594-2596.

Cabungcal, J.H., Steullet, P., Kraftsik, R., Cuenod, M., Do, K.Q., 2012. Early-life insults impair parvalbumin interneurons via oxidative stress: reversal by N-acetylcysteine. Biological Psychiatry. (Epub ahead of print).

Caro, P., Gomez, J., Sanz, A., Portero-Otin, M., Pamplona, R., Barja, G., 2007. Effect of graded corticosterone treatment on aging-related markers of oxidative stress in rat liver mitochondria. Biogerontology 8, 1-11.

Catts, V.S., Catts, S.V., Jablensky, A., Chandler, D., Weickert, C.S., Lavin, M.F., 2012 Evidence of aberrant DNA damage response signalling but normal rates of DNA repair in dividing lymphoblasts from patients with schizophrenia. World Journal of Biological Psychiatry 13, 114-125.

Che, Y., Wang, J.F., Shao, L., Young, T., 2010. Oxidative damage to RNA but not DNA in the hippocampus of patients with major mental illness. Journal of Psychiatry and Neuroscience 35, 296-302.

Chengappa, K.N., Turkin, S.R., Desanti, S., Bowie, C.R., Brar, J.S., Schlicht, P.J., Murphy, S.L., Hetrick, M.L., Bilder, R., Fleet, D., 2012. A preliminary, randomized, double-blind, placebo-controlled trial of $\langle\mathrm{sc}>1<\mid \mathrm{sc}\rangle$-carnosine to improve cognition in schizophrenia. Schizophrenia Research 142, 145-152.

Choy, K.H., Dean, O., Berk, M., Bush, A.I., van den Buuse, M., 2010. Effects of $\mathrm{N}$-acetyl-cysteine treatment on glutathione depletion and a short-term spatial memory deficit in 2-cyclohexene-1-one-treated rats. European Journal of Pharmacology 649, 224-228.

Cohen, S., Williamson, G., 1988. Perceived stress in a probability sample if the United States. In: Spacapan, S, Oskamp, S (Eds.), The Social Psychology of Health: Claremont Symposium on Applied Social Psychology. Sage, Newbury Park, CA. 
Dakhale, G.N., Khanzode, S.D., Khanzode, S.S., Saoji, A., 2005. Supplementation of vitamin $C$ with atypical antipsychotics reduces oxidative stress and improves the outcome of schizophrenia. Psychopharmacology (Berlin) 182, 494-498.

Deng, X.S., Tuo, J., Poulsen, H.E., Loft, S., 1998. Prevention of oxidative DNA damage in rats by brussels sprouts. Free Radical Research 28, 323-333.

Du, J., Wang, Y., Hunter, R., Wei, Y., Blumenthal, R., Falke, C., Khairova, R., Zhou, R., Yuan, P., Machado-Vieira, R., McEwen, B.S., Manji, H.K., 2009. Dynamic regulation of mitochondrial function by glucocorticoids. Proceedings of the National Academy of Sciences of the United States of America 106, 3543-3548.

Epel, E.S., Blackburn, E.H., Lin, J., Dhabhar, F.S., Adler, N.E., Morrow, J.D., Cawthon, R.M., 2004. Accelerated telomere shortening in response to life stress. Proceedings of the National Academy of Sciences of the United States of America 101, $17312-17315$.

Evans, M.D., Olinski, R., Loft, S., Cooke, M.S., 2010a. Toward consensus in the analysis of urinary 8-oxo-7,8-dihydro-2'-deoxyguanosine as a noninvasive biomarker of oxidative stress. FASEB Journal 24, 1249-1260.

Evans, M.D., Saparbaev, M., Cooke, M.S., 2010b. DNA repair and the origins of urinary oxidized 2'-deoxyribonucleosides. Mutagenesis 25, 433-442.

Finkel, T., Holbrook, N.J., 2000. Oxidants, oxidative stress and the biology of ageing. Nature 408, 239-247.

Gysin, R., Kraftsik, R., Sandell, J., Bovet, P., Chappuis, C., Conus, P., Deppen, P. Preisig, M., Ruiz, V., Steullet, P., Tosic, M., Werge, T., Cuenod, M., Do, K.Q., 2007. Impaired glutathione synthesis in schizophrenia: convergent genetic and functional evidence. Proceedings of the National Academy of Sciences of the United States of America 104, 16621-16626.

Harrison, D., Griendling, K.K., Landmesser, U., Hornig, B., Drexler, H., 2003. Role of oxidative stress in atherosclerosis. American Journal of Cardiology 91, 7A-11A

Henriksen, T., Hillestrom, P.R., Poulsen, H.E., Weimann, A., 2009. Automated method for the direct analysis of 8-oxo-guanosine and 8-oxo-2'-deoxyguanosine in human urine using ultraperformance liquid chromatography and tandem mass spectrometry. Free Radical Biology and Medicine 47, 629-635.

Hoang, U., Stewart, R., Goldacre, M., 2011. Mortality after hospital discharge fo people with schizophrenia or bipolar disorder: retrospective study of linked English hospital episode statistics, 1999-2006. British Medical Journal 343, d5422.

Jablensky, A., Angelicheva, D., Donohoe, G.J., Cruickshank, M., Azmanov, D.N. Morris, D.W., McRae, A., Weickert, C.S., Carter, K.W., Chandler, D., Alexandrov, B., Usheva, A., Morar, B., Verbrugghe, P.L., Filipovska, A., Rackham, O., Bishop, A.R., Rasmussen, K.O., Dragovic, M., Cooper, M., Phillips, M., Badcock, J., Bramon-Bosch, E., Almeida, O.P., Flicker, L., Gill, M., Corvin, A., Macgregor, S., Kalaydjieva, L., 2012. Promoter polymorphisms in two overlapping 6 p25 genes implicate mitochondrial proteins in cognitive deficit in schizophrenia. Molecular Psychiatry 17, 1328-1339.

Jeste, D.V., Wolkowitz, O.M., Palmer, B.W., 2011. Divergent trajectories of physical, cognitive, and psychosocial aging in schizophrenia. Schizophrenia Bulletin 37 451-455

Joergensen, A., Broedbaek, K., Weimann, A., Semba, R.D., Ferrucci, L., Joergensen, M.B., Poulsen, H.E., 2011. Association between urinary excretion of cortisol and markers of oxidatively damaged DNA and RNA in humans. PloS One 6 e20795.

Kao, H.T., Cawthon, R.M., Delisi, L.E., Bertisch, H.C., Ji, F., Gordon, D., Li, P., Benedict M.M., Greenberg, W.M., Porton, B., 2008. Rapid telomere erosion in schizophrenia. Molecular Psychiatry 13, 118-119.

Kay, S.R., Fiszbein, A., Opler, L.A., 1987. The positive and negative syndrome scale (PANSS) for schizophrenia. Schizophrenia Bulletin 13, 261-276.

Kirkpatrick, B., Messias, E., Harvey, P.D., Fernandez-Egea, E., Bowie, C.R., 2008. Is schizophrenia a syndrome of accelerated aging? Schizophrenia Bulletin 34 1024-1032.

Kirschbaum, C., Hellhammer, D.H., 1994. Salivary cortisol in psychoneuroendocrine research: recent developments and applications. Psychoneuroendocrinology 19, 313-333.

Kropp, S., Kern, V., Lange, K., Degner, D., Hajak, G., Kornhuber, J., Ruther, E., Emrich, H.M., Schneider, U., Bleich, S., 2005. Oxidative stress during treatment with first- and second-generation antipsychotics. Journal of Neuropsychiatry and Clinical Neurosciences 17, 227-231.

Kunz, M., Gama, C.S., Andreazza, A.C., Salvador, M., Cereser, K.M., Gomes, F.A., Belmonte-de-Abreu, P.S., Berk, M., Kapczinski, F., 2008. Elevated serum superoxide dismutase and thiobarbituric acid reactive substances in differen phases of bipolar disorder and in schizophrenia. Progress in Neuropsychopharmacology and Biological Psychiatry 32, 1677-1681.

Lavoie, S., Murray, M.M., Deppen, P., Knyazeva, M.G., Berk, M., Boulat, O., Bovet, P., Bush, A.I., Conus, P., Copolov, D., Fornari, E., Meuli, R., Solida, A., Vianin, P., Cuenod, M., Buclin, T., Do, K.Q., 2007. Glutathione precursor, N-acetyl-cysteine, improves mismatch negativity in schizophrenia patients. Neuropsychopharmacology 33, 2187-2199.

Loft, S., Larsen, P.N., Rasmussen, A., Fischer-Nielsen, A., Bondesen, S., Kirkegaard, P. Rasmussen, L.S., Ejlersen, E., Tornoe, K., Bergholdt, R., 1995. Oxidative DNA damage after transplantation of the liver and small intestine in pigs. Transplantation 59, 16-20.

Lykkesfeldt, J., 2001. Determination of malondialdehyde as dithiobarbituric acid adduct in biological samples by HPLC with fluorescence detection: comparison with ultraviolet-visible spectrophotometry. Clinical Chemistry 47, 1725-1727.

Lykkesfeldt, J., 2007a. Ascorbate and dehydroascorbic acid as reliable biomarkers of oxidative stress: analytical reproducibility and long-term stability of plasma samples subjected to acidic deproteinization. Cancer Epidemiology, Biomarkers and Prevention 16, 2513-2516.

Lykkesfeldt, J., 2007b. Malondialdehyde as biomarker of oxidative damage to lipids caused by smoking. Clinica Chimica Acta Journal 380, 50-58.

Mansour, H., Chowdari, K., Fathi, W., Elassy, M., Ibrahim, I., Wood, J., Bamne, M., Tobar, S., Yassin, A., Salah, H., Elsayed, H., Eissa, A., El-Boraie, H., Ibrahim, N.E. Elsayed, M., El-Bahaei, W., Gomaa, Z., El-Chennawi, F., Nimgaonkar, V.L., 2011. Does telomere length mediate associations between inbreeding and increased risk for bipolar I disorder and schizophrenia? Psychiatry Research 188 , 129-132.

Miller, G.E., Chen, E., Sze, J., Marin, T., Arevalo, J.M., Doll, R., Ma, R., Cole, S.W., 2008. A functional genomic fingerprint of chronic stress in humans: blunted glucocorticoid and increased NF-kappaB signaling. Biological Psychiatry 64 266-272.

Moller, P., Lohr, M., Folkmann, J.K., Mikkelsen, L., Loft, S., 2010. Aging and oxidatively damaged nuclear DNA in animal organs. Free Radical Biology and Medicine 48, 1275-1285.

Nishioka, N., Arnold, S.E., 2004. Evidence for oxidative DNA damage in the hippocampus of elderly patients with chronic schizophrenia. American Journal of Geriatric Psychiatry 12, 167-175.

Nunomura, A., Hofer, T., Moreira, P.I., Castellani, R.J., Smith, M.A., Perry, G., 2009. RNA oxidation in Alzheimer disease and related neurodegenerative disorders. Acta Neuropathologica 118, 151-166.

Prabakaran, S., Swatton, J.E., Ryan, M.M., Huffaker, S.J., Huang, J.T., Griffin, J.L., Wayland, M., Freeman, T., Dudbridge, F., Lilley, K.S., Karp, N.A., Hester, S., Tkachev, D., Mimmack, M.L., Yolken, R.H., Webster, M.J., Torrey, E.F., Bahn, S., 2004. Mitochondrial dysfunction in schizophrenia: evidence for compromised brain metabolism and oxidative stress. Molecular Psychiatry 9 (684-697), 643.

Pruessner, J.C., Kirschbaum, C., Meinlschmid, G., Hellhammer, D.H., 2003. Two formulas for computation of the area under the curve represent measures of total hormone concentration versus time-dependent change. Psychoneuroendocrinology 28, 916-931.

Psimadas, D., Messini-Nikolaki, N., Zafiropoulou, M., Fortos, A., Tsilimigaki, S., Piperakis, S.M., 2004. DNA damage and repair efficiency in lymphocytes from schizophrenic patients. Cancer Letters 204, 33-40.

Regenold, W.T., Phatak, P., Marano, C.M., Sassan, A., Conley, R.R., Kling, M.A., 2009. Elevated cerebrospinal fluid lactate concentrations in patients with bipolar disorder and schizophrenia: implications for the mitochondrial dysfunction hypothesis. Biological Psychiatry 65, 489-494.

Rosenfeld, M., Brenner-Lavie, H., Ari, S.G., Kavushansky, A., Ben-Shachar, D., 2011. Perturbation in mitochondrial network dynamics and in complex I dependent cellular respiration in schizophrenia. Biological Psychiatry 69, 980-988.

Sahin, E., DePinho, R.A., 2010. Linking functional decline of telomeres, mitochondria and stem cells during ageing. Nature 464, 520-528.

Shenton, M.E., Dickey, C.C., Frumin, M., McCarley, R.W., 2001. A review of MRI findings in schizophrenia. Schizophrenia Research 49, 1-52.

Tosic, M., Ott, J., Barral, S., Bovet, P., Deppen, P., Gheorghita, F., Matthey, M.L., Parnas, J., Preisig, M., Saraga, M., Solida, A., Timm, S., Wang, A.G., Werge, T., Cuenod, M., Do, K.Q., 2006. Schizophrenia and oxidative stress: glutamate cysteine ligase modifier as a susceptibility gene. American Journal of Human Genetics 79, 586-592.

Turrens, J.F., 2003. Mitochondrial formation of reactive oxygen species. Journal of Physiology 552, 335-344.

Velakoulis, D., Wood, S.J., Wong, M.T., McGorry, P.D., Yung, A., Phillips, L., Smith, D., Brewer, W. Proffitt, T., Desmond, P. Pantelis, C., 2006. Hippocampal and amygdala volumes according to psychosis stage and diagnosis: a magnetic resonance imaging study of chronic schizophrenia, first-episode psychosis, and ultra-high-risk individuals. Archives of General Psychiatry 63, 139-149.

Wahlbeck, K., Westman, J., Nordentoft, M., Gissler, M., Laursen, T.M., 2011. Outcomes of Nordic mental health systems: life expectancy of patients with mental disorders. British Journal of Psychiatry 199, 453-458.

Wing, J.K., Babor, T., Brugha, T., Burke, J., Cooper, J.E., Giel, R., Jablenski, A., Regier, D., Sartorius, N., 1990. SCAN. Schedules for Clinical Assessment in Neuropsychiatry. Archives of General Psychiatry 47, 589-593.

Young, J., McKinney, S.B., Ross, B.M., Wahle, K.W., Boyle, S.P., 2007. Biomarkers of oxidative stress in schizophrenic and control subjects. Prostglandines, Leukotrienes and Essential Fatty Acids 76, 73-85.

Zhang, X.Y., Tan, Y.L., Cao, L.Y., Wu, G.Y., Xu, Q., Shen, Y., Zhou, D.F., 2006. Antioxidant enzymes and lipid peroxidation in different forms of schizophrenia treated with typical and atypical antipsychotics. Schizophrenia Research 81, 291-300. 